\title{
A VARIANT OF TCHEBICHEF'S MINIMAX PROBLEM
}

\section{R. J. LEVIT}

1. Introduction. Let $Q_{k}$ be the set of all monic polynomials of degree $k>0$ with real coefficients. Let $\Sigma_{k}$ be the set of all those subsets of the interval $[-1,+1]$ which contain -1 and +1 and have at least one point in each closed subinterval of length $h$. What is the minimum value of $\max _{x \in \sigma}|q(x)|$ as $q$ ranges over $Q_{k}$ while $\sigma$ ranges over $\Sigma_{h}$ ? When $h=0$, the only member of $\Sigma_{h}$ is the entire interval $[-1,+1]$, so that in this case the problem has the classical solution due to Tchebichef,

$$
\max _{x \in \sigma}|q(x)| \geqq \max _{-1 \leqq x \leqq 1}\left|\widetilde{T}_{k}(x)\right|=2^{1-k},
$$

where $\widetilde{T}_{k}(x)$ is the monic Tchebichef polynomial, $\widetilde{T}_{k}(x)=2^{1-k}$. $\cos (k \operatorname{arc} \cos x)$. (See, for example, Natanson [3, Chap. II].) When $k=1,(1-1)$ holds for every $\sigma \in \Sigma_{h}$ independently of the value of $h$; so we henceforth take $k>1$. When $h \geqq 2 /(k-1)$, the problem is trivial, since $\max _{x \in \sigma}|q(x)|$ attains zero as its minimum value for $\sigma=\left\{x_{0}, x_{1}, \cdots, x_{k-1}\right\}, q(x)=\left(x-x_{0}\right)\left(x-x_{1}\right) \cdots\left(x-x_{k-1}\right)$, where $x_{i}=-1+2 i /(k-1)$. There remains the case, $0<h<2 /(k-1)$; and the object of this paper is to construct for any $h$ in this range a polynomial $T_{k}^{(h)}(x)$ in $Q_{k}$ and a subset $\sigma^{*}$ in $\Sigma_{h}$ which together minimize $\max _{x \in \sigma}|g(x)|$ on the product space $Q_{k} \times \Sigma_{h}$. This result is obtained in Theorem 4. The corollary is used in another paper [2] to derive an irreducibility criterion for polynomials over the rational field. The polynomials $T_{\boldsymbol{k}}^{(h)}(x)$ are defined and their relevant properties established in $\$ 3 . ~ \$ 2$ is a preliminary section in which are developed the required properties of certain Hahn polynomials involved in the construction of $T_{k}^{(h)}(x)$.

2. Certain Hahn polynomials. The solution to our problem depends upon the properties of the Hahn polynomials $P_{m}^{(\alpha, \beta, \gamma)}(x)$ with the special parameter values $\alpha= \pm 1 / 2, \beta= \pm 1 / 2$. (All properties of the Hahn polynomials needed here as well as references to other literature on these polynomials can be found in the author's paper [1].) To simplify the notation we define

$$
R_{m}(x)=R_{m}^{(h)}(x)=\frac{(2 h)^{m} m !}{(2 m) !} P_{m}^{(-1 / 2,1 / 2,1 / h)}\left(\frac{x+1}{2 h}\right),
$$

Presented to the Society, April 22, 1967; received by the editors November 1, 1966. 


$$
\begin{aligned}
& \text { (2-2) } S_{m}(x)=S_{m}^{(h)}(x)=\frac{(2 h)^{m} m !}{(2 m) !} P_{m}^{(1 / 2,-1 / 2,1 / h)}\left(\frac{x+1-h}{2 h}\right) \\
& \text { (2-3) } U_{m}(x)=U_{m}^{(h)}(x)=\frac{(2 h)^{m}(m+1) !}{(2 m+1) !} P_{m}^{(1 / 2,-1 / 2,1 / h-1 / 2)}\left(\frac{x+1-h}{2 h}\right), \\
& \text { (2-4) } V_{m}(x)=V_{m}^{(h)}(x)=\frac{(2 h)^{m}(m-1) !}{(2 m-1) !} P_{m}^{(-1 / 2,-1 / 2,1 / h+1 / 2)}\left(\frac{x+1}{2 h}\right),
\end{aligned}
$$

with the understanding that $R_{0}(x)=S_{0}(x)=U_{0}(x)=V_{0}(x)=1$. These are all monic polynomials of degree $m$ with real coefficients. Each of the following formulas can be readily obtained by specializing the indicated formula from [1].

$$
\begin{aligned}
& (x+h-1) R_{m}(x+h)-(x-1) R_{m}(x-h) \\
& =(2 m+1) h S_{m}(x) \text {, } \\
& (x+h+1) S_{m}(x+h)-(x+1) S_{m}(x-h) \\
& =(2 m+1) h R_{m}(x), \\
& (x+h+1)(x+h-1) U_{m-1}(x+h) \\
& -(x+1)(x-1) U_{m-1}(x-h)=2 m h V_{m}(x), \quad[1,(4.3)], \\
& V_{m}(x+h)-V_{m}(x-h)=2 m h U_{m-1}(x), \quad[1,(4.1)] \text {, } \\
& R_{m}(-1)=(-2)^{-m} \prod_{i=1}^{m}(1-i h), \quad[1,(3.0)], \\
& V_{m}(-1)=2(-2)^{-m} \prod_{i=1}^{m}\left[1-\left(i-\frac{1}{2}\right) h\right], \quad[1,(3.0)], \\
& R_{m}(-1-h)=(-2)^{-m} \prod_{i=1}^{m}(1+i h), \\
& V_{m}(-1-h)=2(-2)^{-m} \prod_{i=1}^{m}\left[1+\left(i-\frac{1}{2}\right) h\right], \quad[1,(3.4)] .
\end{aligned}
$$

(2-5) and (2-6) hold for $m \geqq 0$, the rest for $m \geqq 1$.

Next, we establish the formulas,

$$
\begin{aligned}
& (x-1) R_{m}(x) R_{m}(x-h)+C_{2 m+1} \\
& =(x+1) S_{m}(x) S_{m}(x-h)-C_{2 m+1} \text {, } \\
& m \geqq 0,
\end{aligned}
$$

where

(2-13a) $\quad C_{1}=1, \quad C_{2 m+1}=C_{2 m+1}(h)=2^{-2 m} \prod_{i=1}^{m}\left(1-i^{2} h^{2}\right), \quad m \geqq 1$, 


$$
\begin{array}{r}
\left(x^{2}-1\right) U_{m-1}(x) U_{m-1}(x-h)+C_{2 m} \\
=V_{m}(x) V_{m}(x-h)-C_{2 m},
\end{array}
$$

where

$$
C_{2 m}=C_{2 m}(h)=2^{1-2 m} \prod_{i=1}^{m}\left[1-\left(i-\frac{1}{2}\right)^{2} h^{2}\right], \quad m \geqq 1 .
$$

To prove (2-13) we multiply (2-5) through by $R_{m}(x)$ and (2-6) by $S_{m}(x)$ and find that

$$
\begin{aligned}
\Delta_{h}\left[(x-1) R_{m}(x) R_{m}(x-h)\right] & =(2 m+1) h R_{m}(x) S_{m}(x), \\
\Delta_{h}\left[(x+1) S_{m}(x) S_{m}(x-h)\right] & =(2 m+1) h R_{m}(x) S_{m}(x),
\end{aligned}
$$

where $\Delta_{h} f(x)=f(x+h)-f(x)$. The bracketed expressions in the left members are polynomials in $x$, which thus have equal first differences with respect to $x$. Hence, we conclude that

$$
(x+1) S_{m}(x) S_{m}(x-h)-(x-1) R_{m}(x) R_{m}(x-h)=A,
$$

where $A$ is independent of $x$. To evaluate $A$, we set $x=-1$ and find that

$$
A=2 R_{m}(-1) R_{m}(-1-h)=2^{1-2 m} \prod_{i=1}^{m}\left(1-i^{2} h^{2}\right), \quad m \geqq 1,
$$

by $(2-9),(2-11)$, which yields $(2-13) .(2-14)$ is derived similarly by using $(2-7),(2-8)$ to obtain

$$
\begin{aligned}
\Delta_{h}\left[\left(x^{2}-1\right) U_{m-1}(x) U_{m-1}(x-h)\right] & =2 m h U_{m-1}(x) V_{m}(x), \\
\Delta_{h}\left[V_{m}(x) V_{m}(x-h)\right] & =2 m h U_{m-1}(x) V_{m}(x),
\end{aligned}
$$

and then applying (2-10), (2-12).

Next we consider the location and interlacing of the zeros of these polynomials.

Theorem 2-1. Let $0<h<1 / m, m \geqq 1$. Then $R_{m}^{(h)}(x)$ and $S_{m}^{(h)}(x)$ have all real and simple zeros, $r_{1}<r_{2}<\ldots<r_{m}$ and $s_{1}<s_{2}<\ldots<s_{m} r e$ spectively, which are located and spaced as follows:

$$
\begin{aligned}
& -1<r_{i}<1-2 h, \quad-1+h<s_{i}<1-h \\
& (i=1,2, \cdots, m) \\
& r_{i+1}-r_{i}>2 h, \quad s_{i+1}-s_{i}>2 h \\
& (i=1,2, \cdots, m-1), m>1 \text {. }
\end{aligned}
$$

The zeros of $R_{m}^{(h)}(x)$ and $S_{m}^{(h)}(x)$ separate each other. In fact, 


$$
\begin{gathered}
s_{i}-r_{i}>h \quad(i=1,2, \cdots, m) \\
r_{i+1}-s_{i}>h \quad(i=1,2, \cdots, m-1), m>1 .
\end{gathered}
$$

Proof. If $\gamma>m$, the zeros of $P_{m}^{(\alpha, \beta, \gamma)}(x)$ are all real and simple and contained in the open interval $(0, \gamma-1)$ [1, Theorem 1]. As applied to $R_{m}(x)$ and $S_{m}(x)$ this yields $(2-16)$. (2-17) follows from the fact that consecutive zeros of $P_{m}^{(\alpha, \beta, \gamma)}(x)$ are more than one unit apart [1, Theorem 3]. Finally, let $a_{1}<a_{2}<\cdots<a_{m}$ be the zeros of $P_{m}^{(\alpha, \beta, \gamma)}(x)$ and $b_{1}<b_{2}<\cdots<b_{m}$ be the zeros of $P_{m}^{(\alpha-1, \beta+1, \gamma)}(x)$. Then, $a_{i}>b_{i}$ $(i=1,2, \cdots, m)$, and $b_{i+1}>a_{i}+1(i=1,2, \cdots, m-1)$ [1, Theorem 4]. Setting $\alpha=1 / 2, \beta=-1 / 2, \gamma=1 / h$, we have (2-18).

Theorem 2-2. Let $0<h<2 /(2 m-1)$. Then $V_{m}^{(h)}(x)$ for $m \geqq 1$ and $U_{m-1}^{(h)}(x)$ for $m>1$ have all real and simple zeros, $v_{1}<v_{2}<\cdots<v_{m}$ and $u_{1}<u_{2}<\cdots<u_{m-1}$ respectively, which are located and spaced as follows:

$$
\begin{aligned}
&-1+h<u_{i}<1-2 h(i=1,2, \cdots, m-1), \\
&-1<v_{i}<1-h \quad(i=1,2, \cdots, m) \\
& u_{i+1}-u_{i}>2 h \quad(i=1,2, \cdots, m-2), m>2 ; \\
& v_{i+1}-v_{i}>2 h \quad(i=1,2, \cdots, m-1), m>1 .
\end{aligned}
$$

The zeros of $U_{m-1}^{(h)}(x)$ and $V_{m}^{(h)}(x)$ separate each other. In fact,

$$
u_{i}-v_{i}>h, \quad v_{i+1}-u_{i}>h \quad(i=1,2, \cdots, m-1), m>1 .
$$

The proof is the same as in the preceding theorem except that in establishing (2-21) we utilize the result that there is precisely one zero of $P_{m}^{(\alpha+1, \beta+1, \gamma-1)}(x)$ in each interval $\left(a_{i}, a_{i+1}-1\right)$ [1, Theorem 7].

3. The polynomials $T_{k}^{(h)}(x)$. We define the polynomials $T_{k}(x)$ $=T_{k}^{(h)}(x)$ as follows for $k$ odd and even respectively:

$$
\begin{aligned}
T_{2 m+1}^{(h)}(x) & =(x+1) S_{m}^{(h)}(x) S_{m}^{(h)}(x-h)-C_{2 m+1}(h) & & (m \geqq 0), \\
T_{2 m}^{(h)}(x) & =V_{m}^{(h)}(x) V_{m}^{(h)}(x-h)-C_{2 m}(h) & & (m \geqq 1),
\end{aligned}
$$

where $C_{k}(h)$ is defined by $(2-13 a)$ and $(2-14 a) . T_{k}(x)$ is evidently a monic polynomial in $x$ of degree $k>0$ with real coefficients. From (2-13) and (2-14) respectively we have at once

$$
\begin{aligned}
T_{2 m+1}(x) & =(x-1) R_{m}(x) R_{m}(x-h)+C_{2 m+1} & & (m \geqq 0), \\
T_{2 m}(x) & =\left(x^{2}-1\right) U_{m-1}(x) U_{m-1}(x-h)+C_{2 m} & & (m \geqq 1) .
\end{aligned}
$$

Next we prove the following pair of theorems. 
THEOREM 3-1. Let $0<h<1 / m, m \geqq 1$. In the notation of Theorem 2-1,

$$
T_{2 m+1}(x) \geqq C_{2 m+1}
$$

when and only when

$$
x \geqq 1 \text { or } r_{i} \leqq x \leqq r_{i}+h \quad \text { for any } i .
$$

Also,

$$
T_{2 m+1}(x) \leqq-C_{2 m+1}
$$

when and only when

$$
x \leqq-1 \text { or } s_{i} \leqq x \leqq s_{i}+h \quad \text { for any } i .
$$

TheOREM 3-2. Let $0<h<2 /(2 m-1), m \geqq 1$. In the notation of Theorem 2-2,

$$
T_{2 m}(x) \geqq C_{2 m}
$$

when and only when

$$
|x| \geqq 1 \text { or (when } m>1) \quad u_{i} \leqq x \leqq u_{i}+h \quad \text { for any } i \text {. }
$$

Also,

$$
T_{2 m}(x) \leqq-C_{2 m}
$$

when and only when

$$
v_{i} \leqq x \leqq v_{i}+h \quad \text { for any } i .
$$

Equality holds in (3-5a) if and only if it holds in (3-5b), and similarly for (3-6), (3-7), (3-8). We give the proof for (3-7). The other three cases do not differ significantly. From (3-4) it is clear that $T_{2 m}(x)=C_{2 m}$ when $x= \pm 1$ and when $x=u_{i}$ and $x=u_{i}+h$ $(i=1,2, \cdots, m-1)$. Let $x_{1}>1$. In view of $(2-19)$, there is no zero of $U_{m-1}(x)$ in the interval, $x_{1}-h \leqq x \leqq x_{1}$. Consequently, $U_{m-1}\left(x_{1}-h\right)$ and $U_{m-1}\left(x_{1}\right)$ have the same sign, and so, by (3-4), $T_{2 m}\left(x_{1}\right)>C_{2 m}$. Similarly, for a point $x_{2}<-1, T_{2 m}\left(x_{2}\right)>C_{2 m}$. Next, consider a point $x_{3}$ such that for some zero $u_{i}$ of $U_{m-1}(x)$,

$$
u_{i} \leqq x_{3} \leqq u_{i}+h .
$$

Then by $(2-19),-1<x_{3}<1$, so that

$$
x_{3}^{2}-1<0 .
$$

By (3-9), $U_{m-1}(x)$ has the zero $u_{i}$ in the interval $\left[x_{3}-h, x_{3}\right]$. By (2-20), this is the only zero in that interval. Hence, $U_{m-1}\left(x_{3}-h\right)$ and $U_{m-1}\left(x_{3}\right)$ 
have opposite signs or one of them vanishes. Combining this fact with (3-10), we conclude that $T_{2 m}\left(x_{3}\right) \geqq C_{2 m}$ also. Conversely, suppose that $T_{2 m}\left(x_{0}\right) \geqq C_{2 m}$ for some point $x_{0}$ inside $(-1,1)$. Then by $(3-4)$, $U_{m-1}\left(x_{0}\right)$ and $U_{m-1}\left(x_{0}-h\right)$ must have opposite signs or one of them must vanish. Hence, $U_{m-1}(x)$ has a zero $u_{i}$ such that $x_{0}-h \leqq u_{i} \leqq x_{1}$. But then, $u_{i} \leqq x_{0} \leqq u_{i}+h$, which completes the proof.

Next, we note that by (3-1), (2-15.1) and by (3-2), (2-15.2),

$$
\begin{aligned}
\Delta_{h} T_{2 m+1}(x) & =(2 m+1) h R_{m}(x) S_{m}(x), \\
\Delta_{h} T_{2 m}(x) & =2 m h U_{m-1}(x) V_{m}(x) .
\end{aligned}
$$

From the second of these we find by $(2-7),(2-8)$ and (3-2), (3-4) that

$$
\begin{aligned}
& (x+h+1)(x+h-1) \Delta_{h} T_{2 m}(x+h) \\
& \quad=4 m^{2} h^{2} \Delta_{h} T_{2 m}(x)+\left(x^{2}-1\right) \Delta_{h} T_{2 m}(x-h)+8 m^{2} h^{2} T_{2 m}(x) .
\end{aligned}
$$

This establishes the difference equation,

$$
\begin{aligned}
(x+2 h+1)( & +2 h-1) \Delta_{h}^{3} T_{k}(x) \\
& +\left[2(x+h+1)(x+h-1)-k^{2} h^{2}\right] \Delta_{h}^{2} T_{k}(x) \\
& +\left(2 x+3 h-3 k^{2} h\right) h \Delta_{h} T_{k}(x)-2 k^{2} h^{2} T_{k}(x)=0,
\end{aligned}
$$

for $k=2 m$. This equation holds for $k=2 m+1$ as well. The proof in that case utilizes the first of equations (3-11) and (2-5), (2-6), (3-1), (3-3).

4. The minimax problem. We are now ready to establish the minimax property of the polynomials $T_{k}^{(h)}(x)$.

THEOREM 4. Let $\Sigma_{h}$ be the set of all subsets of the interval $[-1,+1]$ which contain the endpoints and at least one point in each closed subinterval of length $h$. Let $Q_{k}$ be the set of all monic polynomials of degree $k>1$ with real coefficients. If

$$
0<h<2 /(k-1),
$$

then there is a set $\sigma^{*}$ in $\Sigma_{h}$ such that, for every polynomial $q$ in $Q_{k}$ and every set $\sigma$ in $\Sigma_{h}$,

$$
\begin{aligned}
& \max _{x \in \sigma}|q(x)| \geqq \max _{x \in \sigma^{*}}\left|T_{k}^{(h)}(x)\right| \\
&=C_{k}(h)=2^{1-k} \prod_{i=1}^{k}\left\{1+\left(i-\frac{k+1}{2}\right) h\right\} .
\end{aligned}
$$

Proof. We note that the expression for $C_{k}(h)$ in $(4-2)$ reduces to 
(2-13a) when $k$ is odd and to (2-14a) when $k$ is even. In order to construct a set $\sigma^{*}$ such that $\max _{x \in \sigma^{*}}\left|T_{k}^{(h)}(x)\right|=C_{k}$, we form the set $\sigma_{0}$ consisting of $-1,+1$, and all points $n h$ for integral $n$ such that $-1<n h<1$. Clearly, $\sigma_{0}$ is in $\Sigma_{h}$. From $\sigma_{0}$ we construct $\sigma^{*}$ for $k$ even by replacing each point $n h$ falling in an interval $\left(u_{i}, u_{i}+h\right)$ or $\left(v_{i}, v_{i}+h\right)$ (notation of Theorem 2-2) by both endpoints of the interval. No ambiguity can arise as to which interval a given point lies in, since by (2-20), (2-21) no two of these in tervals overlap. For $k$ odd, $\sigma^{*}$ is obtained from $\sigma_{0}$ by replacing points $n h$ falling in intervals $\left(r_{i}, r_{i}+h\right)$ or $\left(s_{i}, s_{i}+h\right)$ by both endpoints of the interval. In this case, absence of overlap between intervals is assured by (2-17), (2-18). Since these replacements can only reduce the distance between consecutive points, $\sigma^{*}$ also belongs to $\Sigma_{h}$. It follows from (4-1) and Theorem 3-1 or 3-2 that $\left|T_{k}(x)\right| \leqq C_{k}$ for all $x$ in $\sigma^{*}$ with equality holding for $x= \pm 1$ (and possibly other points of $\sigma^{*}$ ). Hence, $\max _{x \in \sigma^{*}}\left|T_{k}(x)\right|=C_{k}$.

Next, consider any set $\sigma$ in $\Sigma_{h}$. By (2-16) and (2-19) there is at least one point of $\sigma$ in each of the intervals, $\left[r_{i}, r_{i}+h\right],\left[s_{i}, s_{i}+h\right]\left[u_{i}, u_{i}+h\right]$, $\left[v_{i}, v_{i}+h\right]$. We select $k+1$ points from $\sigma$ as follows: $x_{0}=-1, x_{k}=+1$; for $k=2 m, x_{2 i}$ is any point of $\sigma$ in $\left[u_{i}, u_{i}+h\right](i=1,2, \cdots, m-1)$, and $x_{2 i-1}$ is any point of $\sigma$ in $\left[v_{i}, v_{i}+h\right](i=1,2, \cdots, m)$; for $k=2 m$ $+1, x_{2 i}$ is any point of $\sigma$ in $\left[s_{i}, s_{i}+h\right]$, and $x_{2 i-1}$ is any point of $\sigma$ in $\left[r_{i}, r_{i}+h\right](i=1,2, \cdots, m)$. By Theorem 2-1 or 2-2,

$$
-1=x_{0}<x_{1}<\cdots<x_{k}=+1 .
$$

By (4-1) and Theorem 3-1 or 3-2,

$$
\begin{array}{ll}
T_{k}\left(x_{j}\right) \geqq C_{k} & \text { for } j \equiv k(\bmod 2), \\
T_{k}\left(x_{j}\right) \leqq-C_{k} & \text { for } j \neq k=(\bmod 2) .
\end{array}
$$

Now let $q(x)$ be any polynomial in $Q_{k}$, and suppose that

$$
\left|q\left(x_{j}\right)\right|<C_{k}, \quad(j=0,1, \cdots, k) .
$$

Then by (4-4), $T_{k}\left(x_{j}\right)-q\left(x_{j}\right)$ would have the sign of $T_{k}\left(x_{j}\right)$-namely, positive for $j \equiv k(\bmod 2)$ and negative for $j \not \equiv k(\bmod 2)$. Hence by (4-3), $T_{k}(x)-q(x)$ changes sign $k$ times in the interval $[-1,+1]$. But this is impossible, since $T_{k}-q$ is a polynomial of degree less than $k$. Consequently, (4-5) is false; so we have

$$
\max _{x \in \sigma}|q(x)| \geqq \max _{\mathcal{J}=0,1, \ldots, k}\left|q\left(x_{j}\right)\right| \geqq C_{k},
$$

and the proof is complete.

As pointed out by the referee, the proof may be slightly modified 
to show that strict inequality holds in (4-2) unless $g$ is $T_{k}^{(h)}$. Instead of (4-5) we suppose that

$$
|q(x)| \leqq C_{k}, \quad(j=0,1, \cdots, k) .
$$

Our conclusion then is that $T_{k}(x)-q(x)$ has at least $k$ zeros (not necessarily distinct) in $[-1,+1]$ and hence vanishes identically. On the other hand, $\sigma^{*}$ is not.unique. For $\sigma^{*}$ in $\Sigma_{h}, \max _{x \in \sigma^{*}}\left|T_{k}(x)\right|=C_{k}$ if and only if $\sigma^{*}$ contains both endpoints but no other points of each interval $\left[u_{i}, u_{i}+h\right]$ and $\left[v_{i}, v_{i}+h\right]$ for $k$ even, $\left[r_{i}, r_{i}+h\right]$ and $\left[s_{i}, s_{i}+h\right]$ for $k$ odd.

Corollary 4. Let $c_{1}<c_{2}<\cdots<c_{n}$ be real numbers, let $L=c_{n}-c_{1}$ and $d=\max \left(c_{i+1}-c_{i}\right)$, and let $q(x)$ be any polynomial in $Q_{k}$. If $L>d(k-1)$,

$$
\max _{i=1,2, \ldots, n}\left|q\left(c_{i}\right)\right| \geqq 2^{1-2 k} \prod_{i=1}^{k}\{L+d(2 i-1-k)\} .
$$

Proof. Let $y_{i}=\left(2 c_{i}-c_{1}-c_{n}\right) / L$, and define the polynomial $p(y)$ $=(2 / L)^{k} q\left(c_{1}+(L / 2)(y+1)\right)$. Then, $\left\{y_{1}, y_{2}, \cdots, y_{n}\right\}$ belongs to $\sum_{2 d} / L$, and $p(y)$ belongs to $Q_{k}$. Since $q\left(c_{i}\right)=(L / 2)^{k} p\left(y_{i}\right)$, Theorem 4-1 yields

$$
\max _{i=1,2, \ldots, n}\left|q\left(c_{i}\right)\right| \geqq(L / 2)^{k} C_{k}(2 d / L)
$$

\section{REFERENCES}

1. R. J. Levit, The zeros of the Hahn polynomials, SIAM Rev. 9 (1967), 191-203. 2. - Irreducibility of polynomials with low absolute values, (to appear).

3. I. P. Natanson, Constructive function theory, Vol. 1, Ungar, New York, 1964. San Francisco State College 